PROCEEDINGS OF THE WORLD CONFERENCE ON OZONE THERAPY IN MEDICINE, DENTISTRY AND VETERINARY. ANCONA (ITALY). SEPTEMBER 22nd - 23rd - 24th, 2017

\title{
Hashimoto's Chronic Thyroiditis treated with Systemic Ozone Therapy [abstract]
}

\author{
Frank Hernandez-Rosales, Yaisy Picrin-Duany
}

Health Integral Clinic Dr. Yaisy Picrin, Santiago de Chile

\section{ABSTRACT}

\section{OPEN ACCESS}

\section{Citation \\ Hernandez-Rosales F, Picrin-Duany Y. Hashimoto's Chronic Thyroiditis treated with Systemic Ozone Therapy [abstract]. Proceedings of The World Conference on Ozone Therapy in Medicine, Dentistry and Veterinary. Ancona (Italy). September 22nd - 23rd - 24th , 2017. J Ozone Ther. 2019;3(4):51. doi: 10.7203/ jo3t.3.4.2019.15531}

Academic Editor Jose Baeza-Noci,

School of Medicine, Valencia University, SPAIN

\section{Editor}

World Federation of Ozone Therapy, Bolgna, ITALY

\section{Received}

June 17, 2019

\section{Accepted}

December 08, 2019

Published

December 30, 2019

Intellectual Property

Frank Hernandez-Rosales.

This is an open access article distributed under the terms of the Creative Commons Attribution License (CC BY 4.0), which permits unrestricted use, distribution, and reproduction in any medium, provided the original author and source are credited.

\section{Author Information}

frankozono@yahoo.com
Purpose. This cross-sectional investigation was done to evaluate the effectiveness of ozone therapy to regulate the oxidative stress associated with chronic Hashimoto's thyroiditis (HT).

Material and Methods. Diagnosed patients with chronic HT and on thyroxine therapy were recruited for additional treatment with rectal ozone therapy. Thyroid stimulating hormone (TSH), thyroid hormones (fT4 and T3), anti-thyroidperoxidase antibodies (anti-TPO), anti-thyroidglobulin antibodies (anti-Tg) were assessed. Oxidative stress (OS) diagnosis was assessed by a specific system including a computer program which gives 4 parameters (total antioxidants, total prooxidants, redox index (RI), and grade) from the results of 8 blood biomarkers (GSH, GPx, SOD, CAT, CD, MAD, THP, AOPP).

Results. Before treatment with ozone therapy, mean of TSH, fT4, and T3 showed values within the reference ranges, but not corresponding to antiTPO and anti-Tg levels, which presented high ones. Initial RI was in a range of values corresponding to degrees of oxidative stress from light to severe. After 3 cycles of treatment with ozone therapy the average RI increased significantly, so the degrees of OS were from moderate to very light. Mainly GSH was the most affected marker in antioxidant defense deficiency. Thyroid function markers were significantly better than previous numbers, whereas antithyroid antibodies decreased their titre not significantly in most patients.

Discussion. OS has been implicated in the pathogenesis of several inflammatory and immune-mediated disorders including HT. Enhanced OS seems to be mainly related to the chronic autoimmune inflammation rather than to variations of intracellular thyroid hormone. The systemic ozone therapy has the property to regulate the OS effectively; therefore, the application of this therapy in HT can be of great importance for the control of the disease.

Conclusion. The present preliminary results suggest that systemic ozone therapy may be helpful in providing protection against OS associated with this disease. However, further long-term treatment studies are needed to ascertain the effectiveness of this therapy on disorders in the immune system of patients with HT. 